\title{
Bacterial magnetosomes-based nanocarriers for co-delivery of cancer therapeutics in vitro
}

This article was published in the following Dove Press journal:

International Journal of Nanomedicine

\author{
Rui-Min Long ${ }^{1,2, *}$ \\ Qing-Lei Dai ${ }^{1, *}$ \\ Xia Zhou',* \\ Duan-Hua Cai' \\ Ya-Zhen Hong ${ }^{1-3}$ \\ Shi-Bin Wang ${ }^{1-4}$ \\ Yuan-Gang Liu'-3
}

'College of Chemical Engineering, Huaqiao University, Xiamen 36I02I, China; ${ }^{2}$ Fujian Provincial Key

Laboratory of Biochemical Technology, Huaqiao University, Xiamen 36I02I,

China; ${ }^{3}$ Institute of Pharmaceutical

Engineering, Huaqiao University,

Xiamen 36102I, China; ${ }^{4}$ Institute of

Biomaterials and Tissue Engineering, Huaqiao University, Xiamen 36I02I, China

*These authors contributed equally to this work
Correspondence: Yuan-Gang Liu Institute of Pharmaceutical Engineering, College of Chemical Engineering, Huaqiao University, No 668 Jimei Avenue, Xiamen 361021, China

Tel +86 I52 5926 I2I6

Email ygliu@hqu.edu.cn

\begin{abstract}
In recent times, co-delivery of therapeutics has emerged as a promising strategy for treating dreadful diseases such as cancer.

Materials and methods: In this study, we developed a novel nanocarrier based on bacterial magnetosomes (BMs) that co-loaded with siRNA and doxorubicin (DOX) using polyethyleneimine (PEI) as a cross-linker (BMs/DP/siRNA). The delivery efficiency of siRNA as well as the $\mathrm{pH}$-responsive release of DOX, and synergistic efficacy of these therapeutics in vitro were systematically investigated.
\end{abstract}

Results: The structure of DOX-PEI(DP) conjugates that synthesized via hydrazone bond formation was confirmed by ${ }^{1} \mathrm{H}$ nuclear magnetic resonance (NMR). The in vitro release experiments showed that the DP conjugate (DOX-loading efficiency $-5.77 \% \pm 0.08 \%$ ) exhibited the long-term release behavior. Furthermore, the optimal BMs/DP/siRNA particle size of $107.2 \mathrm{~nm}$ and the zeta potential value of $31.1 \pm 1.0 \mathrm{mV}$ facilitated enhanced cellular internalization efficiency. Moreover, the agarose gel electrophoresis showed that the co-delivery system could protect siRNA from degradation in serum and RNase A. In addition, the cytotoxicity assay showed that BMs/DP/siRNA could achieve an excellent synergistic effect compared to that of siRNA delivery alone. The acridine orange (AO)/ ethidium bromide (EB) double staining assay also showed that BMs/DP/siRNA complex could induce cells in a stage of late apoptosis and nanocomplex located in the proximity of the nucleus. Conclusion: The combination of gene and chemotherapeutic drug using BMs is highly efficient, and the $\mathrm{BMs} / \mathrm{DP} / \mathrm{siRNA}$ would be a promising therapeutic strategy for the future therapeutics. Keywords: bacterial magnetosomes, co-delivery, gene therapy, $\mathrm{pH}$-responsive release

\section{Introduction}

Currently, the co-delivery of therapeutics that particularly includes the combination of nucleic acids and chemotherapeutic drugs has attracted enormous interest due to their enormous therapeutic efficacy over those delivered solely. In addition, this strategy offers numerous advantages such as the use of limited doses of cytotoxic agents resulting in the reduced side effects, delay in the development of cancer drug resistance, leading to augmented intracellular drug levels, and could deliver multiple therapeutic agents that act through different antiproliferative mechanisms. ${ }^{1,2}$ Therefore, an ideal carrier platform is required to protect nucleic acid from degradation and enable to control the release of the gene as well as chemotherapeutic agent, for synergistic therapeutic efficacy.

In recent years, considerable efforts have been put forward to construct the co-delivery systems. For example, the co-delivery of doxorubicin (DOX) and valproic acid could result in the internalization of latter into HepG2 cell line when co-delivered illustrating efficient combination therapy. ${ }^{3}$ Moreover, the nanocarriers including liposomes, ${ }^{4,5}$ polymers, ${ }^{6-9}$ and several inorganic materials such as mesoporous silica nanoparticles and layered double hydroxides ${ }^{10-13}$ have been used for delivering 
genes and drugs. ${ }^{14}$ Among them, polyethyleneimine (PEI), a cationic polymer, has been widely used for gene delivery due to its advantages of highly positive charge and abundant amine groups. ${ }^{15-17}$ In this framework, chemotherapeutic drug conjugates with the cationic polymer via $\mathrm{pH}$-sensitive linker followed by binding with gene through electrostatic interactions, which can achieve an effective combinatorial delivery of nucleic acid and chemotherapeutic drug. ${ }^{18}$ The obtained delivery system can not only avoid enzyme degradation but also control the release rate of therapeutic drugs.

Bacterial magnetosomes (BMs) have emerged as a novel and efficient material due to their advantages of excellent biocompatibility and abundant bioactive sites in the membrane. ${ }^{19,20} \mathrm{BMs}$ are membrane-covered nanocrystals of magnetic iron oxide or iron sulfide synthesized by magnetotactic bacteria. ${ }^{21}$ The advantageous properties of them make BMs a prior candidate for the delivery of proteins, chemotherapeutic drugs, and genes, among others. ${ }^{22-24}$ Compared to synthetic magnetic nanoparticles, BMs offer various advantages, such as cost-effective, ease of synthesis, and surface modification, which make them more promising to become the nanocarrier for the co-delivery of therapeutics. ${ }^{25}$

Here, we designed an efficient co-delivery platform based on siRNA- and DOX-loaded BMs using PEI as a cross-linker (BMs/DP/siRNA) for cancer therapeutics. First, the bifunctional succinimidyl 6-hydrazinonicotinate acetone hydrazine (SANH) was selected as a cross-linking agent to conjugate DOX and PEI. Then, DOX-PEI (DP) conjugate was synthesized, following being condensed the anionic siRNA and charged with negatively charged $\mathrm{BMs}$, resulting in the construction of BMs/DP/siRNA. Furthermore, the release of various therapeutic agents in vitro and their synergistic antitumor efficacy were systematically investigated.

\section{Materials and methods Materials}

BMs obtained from Magnetospirillum gryphiswaldense MSR-1 were a gift from Professor Li Ying and Jiang Wei (Department of Microbiology, China Agricultural University). DOX $\cdot \mathrm{HCl}$ was purchased from Dalian Meilun Biology Technology Co., Ltd. (Shanghai, China). SANH was obtained from Dernopharm Co., Ltd. (Nanjing, China). Branched PEI (molecular weight: $25 \mathrm{kDa}$ ) was purchased from Sigma-Aldrich Co., (St Louis, MO, USA). STAT 3 siRNA and siRNA (NC) were obtained from GenePharma Co., Ltd. (Shanghai, China), and siRNA (NC) was the negative control of STAT 3 siRNA without homology. The
HeLa cervical carcinoma cell line was from Typical China Academy Culture Preservation Committee Cell Library (Shanghai, China). Cell culture medium was composed of DMEM supplemented with $10 \%$ FBS. All cells were incubated at $37^{\circ} \mathrm{C}$ in humidified air with $5 \% \mathrm{CO}_{2}$.

\section{Synthesis and characterization of DOX-PEI conjugates}

The synthesis of DOX-PEI conjugates was carried out in two steps (Figure 1). First, SANH-PEI was conjugated by the reaction of $N$ - $N$-hydroxysuccinimide (NHS) groups of SANH with the amine groups of PEI. Briefly, SANH was initially dissolved in dimethyl sulfoxide and PEI was then added to the mixture. Following that, the mixture was added to the modified buffer $(0.877 \%$ sodium chloride, $1.639 \%$ sodium phosphate, $\mathrm{pH} 7.4$ ) and stirred for $48 \mathrm{~h}$ under nitrogen atmosphere. The resultant was then purified by dialysis (molecular weight: 8,000-14,000). After lyophilizing, the product was confirmed by ${ }^{1} \mathrm{H}$ nuclear magnetic resonance (NMR) ( $\left.\mathrm{CD}_{3} \mathrm{SOCD}_{3}, 400 \mathrm{MHZ}\right)$. Second, DOX-PEI was obtained by the reaction between ketone groups of DOX and hydrazine groups of SANH-PEI resulting in the hydrazone bond formation. DOX and SANH-PEI were dissolved in conjugation buffer $(0.877 \%$ sodium chloride, $1.639 \%$ sodium phosphate, $\mathrm{pH}$ 6.0) and stirred for $48 \mathrm{~h}$ in the dark under nitrogen supply. The product was recovered after purification by dialysis followed by lyophilization. Then, ${ }^{1} \mathrm{H}$ NMR $\left(\mathrm{CD}_{3} \mathrm{SOCD}_{3}, 400 \mathrm{MHZ}\right)$ was used to confirm the structure of DOX-PEI conjugate. The amount of DOX in DOX-PEI conjugate was determined by UV-vis spectrophotometry at $481 \mathrm{~nm}$. The method for the determination of DOX content was in accordance with the validation requirements. In the determination process, we ensured that PEI and SANH had no interference with DOX. In pH 7.4 and 5.5 PBS buffer, the standard curve of DOX was $Y=0.0200 X+0.0033\left(R^{2}=0.9995\right)$ and $Y=0.0173 X+0.0027\left(R^{2}=0.9997\right)$, respectively.

\section{$\mathrm{pH}$-sensitive release of DOX}

The $\mathrm{pH}$-sensitive release of DOX from DP was determined in PBS buffer that was adjusted to $\mathrm{pH}$ of 5.5 and 7.4. Briefly, DP was dissolved in $1 \mathrm{~mL}$ of PBS and placed in the dialysis bag. Following that, the dialysis bag was placed in the tube containing $20 \mathrm{~mL}$ of PBS at different $\mathrm{pH}$ values. Then, the release process was performed by placing the bags in the incubator that maintained at $37^{\circ} \mathrm{C}$ and $60 \mathrm{rpm}$. The released amount of DOX in the solution was measured by using UV-vis spectrophotometry after incubation for $0.5,1,2,4,8$, and $12 \mathrm{~h}$. All assays were performed in triplicate. 

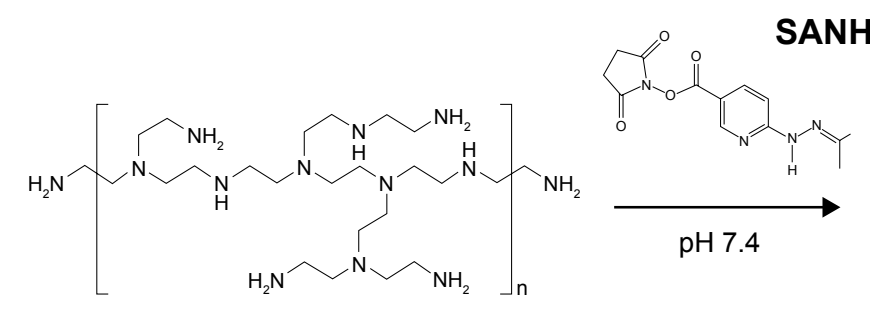

SANH

PEI

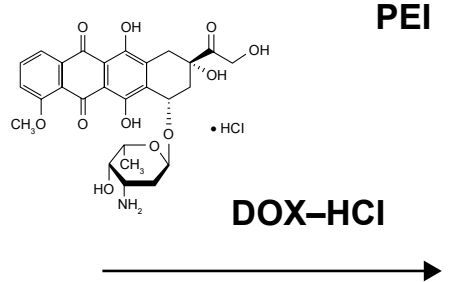

pH 6.0
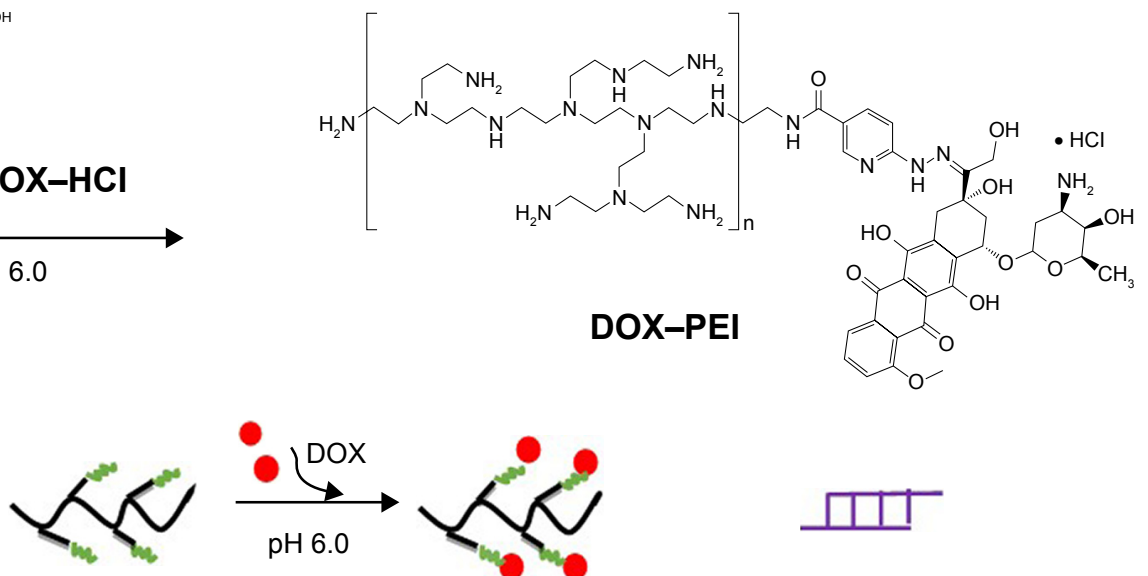

SANH-PEI

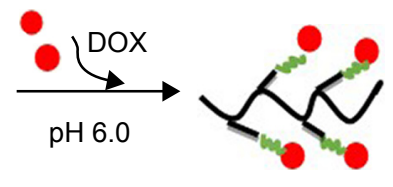

DP

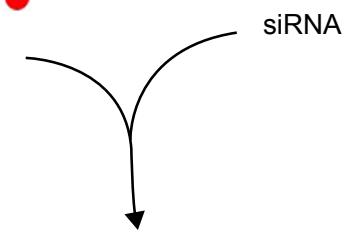

Mixed with siRNA

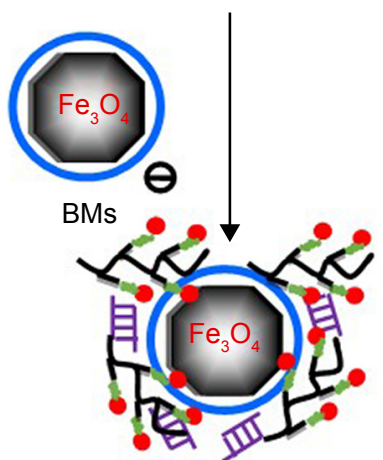

BMs/DP/siRNA

Figure I Fabrication of BMs/DP/siRNA nanocomplexes.

Abbreviations: BMs, bacterial magnetosomes; DOX, doxorubicin; DP, DOX-PEI; PEI, polyethyleneimine; SANH, succinimidyl 6-hydrazinonicotinate acetone hydrazine.

\section{Preparation and characterization of BMs/ DP/siRNA nanocomplexes}

$\mathrm{BMs} / \mathrm{DP} / \mathrm{siRNA}$ nanocomplexes were synthesized as shown in Figure 1. The nanocomplexes with different DP/siRNA weight ratios (ratio was set as $0,0.13,0.26,0.52,1.03,1.29$, 2.06 , and 2.58 ) were prepared by adding appropriate amount of DP into siRNA-dispersed diethyl pyrocarbonate (DEPC) water, following that a fixed amount of BMs was added (BMs/ siRNA weight ratio was 1:2). The mixture was vortexed for
$2 \mathrm{~min}$ and incubated for $25 \mathrm{~min}$ at room temperature and collected. The formation of composites was determined by the gel electrophoresis assay. Briefly, the composites were added to $0.8 \%(\mathrm{w} / \mathrm{v})$ agarose gel containing $1 \%(\mathrm{v} / \mathrm{v})$ gel stain in tris acetate EDTA (TAE) buffer, the gel was run at $70 \mathrm{~V}$ for $20 \mathrm{~min}$ and imaged under UV transilluminator and a digital imaging system. The particle size and zeta potential of BMs/DP/siRNA were characterized by Zetasizer (ZEN3600; Malvern Instruments, Malvern, UK) in DEPC water. 
To investigate whether the concentration of BMs affected the eventual formulation, BMs were treated with the DP/ siRNA (DP/siRNA =2.06) complexes in DEPC water and incubated for $25 \mathrm{~min}$ (BMs/siRNA weight ratio was set as $1: 5,1: 2,1: 1,2: 1$, and $5: 1)$. The products were characterized by agarose gel retardation assay and Zetasizer (ZEN3600).

\section{Stability of BMs/DP/siRNA nanocomplexes}

For heparin decomplexation assay, the prepared BMs/DP/ siRNA nanocomplexes were mixed with heparin (heparin/ siRNA weight ratio was 2, 10, 25, and 100) and incubated for $15 \mathrm{~min}$. The resultants were subjected to agarose gel electrophoresis as described earlier.

For serum stability assay, after adding 50\% FBS, naked siRNA and BMs/DP/siRNA nanocomplexes were incubated for $60,90,120$, and $150 \mathrm{~min}$. At predetermined intervals, the mixture was treated with heparin followed by incubation for a further $15 \mathrm{~min}$. All samples were loaded on agarose gel for retardation analysis.

For enzyme stability assay, the procedure was the same as the serum stability assay, but the only difference was that naked siRNA and BMs/DP/siRNA were incubated with RNase A.

\section{Cell viability assay}

To assess the synergistic antitumor effect of BMs/DP/siRNA complexes, the cytotoxicity with different siRNA contents and incubation times was determined using the cell counting kit-8 (CCK-8) assay. HeLa cells were seeded on 96-well plates at a density of $2 \times 10^{4}$ cells/well and incubated for $24 \mathrm{~h}$ for proper cell attachment. Then, the media were replaced with siRNA, DOX, BMs-PEI/SANH, BMs-PEI/SANHsiRNA (STAT 3), BMs-PEI/SANH-siRNA (NC), and BMs/ DP/siRNA (STAT 3) (the dosage of siRNA was set as 2.5, $5,7.5$, and $10 \mathrm{pmol}$, and the DP/siRNA ratio was $1.03,1.29$, 2.06, and 2.58) in $100 \mu \mathrm{L}$ of serum-free DMEM for $6 \mathrm{~h}$ to protect siRNA from protein damage in serum and then followed by $200 \mu \mathrm{L}$ of DMEM containing 10\% FBS for further $48 \mathrm{~h}$. Subsequently, the medium was treated with a CCK-8 working solution and incubated for additional $2 \mathrm{~h} .{ }^{26}$ The absorption at $450 \mathrm{~nm}$ was performed using a microplate reader.

Cytotoxicity assay with different incubation times was evaluated as described earlier. Briefly, siRNA, DOX, BMsPEI/SANH, BMs-PEI/SANH-siRNA (STAT 3), BMs-PEI/ SANH-siRNA (NC), and BMs/DP/siRNA (STAT 3) (the dosage of siRNA was $7.5 \mathrm{pmol}$ ) were incubated with HeLa cells in $100 \mu \mathrm{L}$ of serum-free DMEM for $6 \mathrm{~h}$ followed by $200 \mu \mathrm{L}$ of DMEM containing 10\% FBS for further 24, 48, and $72 \mathrm{~h}$. Then, the cell viability was measured by repeating the abovementioned procedure.

\section{Cell apoptosis assay}

HeLa cells were seeded on 24 -well plates at $1 \times 10^{5}$ cells/well and incubated for $24 \mathrm{~h}$. Then, the cells were incubated with siRNA, DOX, BMs-PEI/SANH-siRNA, and BMs/DP/ siRNA (the concentration of siRNA was $50 \mathrm{nM}$ ) in $0.5 \mathrm{~mL}$ of serum-free DMEM for $6 \mathrm{~h}$ followed by $48 \mathrm{~h}$ in $1 \mathrm{~mL}$ DMEM containing $10 \%$ FBS. Then, the harvested cells were washed three times with $\mathrm{PBS}$, after being stained with acridine orange (AO)/ethidium bromide (EB) mixed dyes reagent for 2-3 min in the dark. Finally, the cell apoptosis was analyzed using a confocal laser scanning microscope (CLSM, TCS SP8; Leica Microsystems, Wetzlar, Germany).

\section{Cellular uptake of BMs/DP/siRNA nanocomplexes}

HeLa cells at a density of $4 \times 10^{5}$ cells/well were seeded in $35 \mathrm{~mm}$ glass-bottom dishes and incubated for $24 \mathrm{~h}$. Then, the FAM-labeled BMs/DP/siRNA nanocomplexes were incubated with HeLa cells (the concentration of siRNA was $50 \mathrm{nM}$ ) according to the protocol of staining with mixed dye reagent (dye reagent 1 and dye reagent 2) for 2-3 $\mathrm{min}$. At 0.5 and $6 \mathrm{~h}$, the cells were washed thrice with cold PBS and fixed with $4 \%$ formaldehyde for $10 \mathrm{~min}$ and stained with DAPI for 10 min with intermittent PBS washes. Finally, the intracellular distribution of nanocomplexes was observed under CLSM.

\section{Results and discussion Synthesis and characterization of DOX-PEI conjugate}

Recently, numerous studies have reported that the hydrazone bond as a $\mathrm{pH}$-sensitive linkage, which could effectively deliver a chemotherapeutic agent such as DOX in tumor environment. ${ }^{27}$ While the commonly applied methods for fabricating hydrazone bonds involved complex reaction processes that utilize organic reagents and harsh conditions. ${ }^{28}$ To overcome these issues, here we used a bifunctional crosslinker containing an NHS section to incorporate PEI and a hydrazine section to conjugate with DOX. This synthesis process was simple and mild with excellent biocompatibility attribute. SANH has been an increasing interest to harness the conjugation of proteins, polypeptides, and DNA, while there are few studies on drug conjugation for its delivery. ${ }^{29,30}$

The freeze-dried resultant DP conjugate was confirmed by ${ }^{1} \mathrm{H}$ NMR (Figure 2). From the spectrum of DP, the 


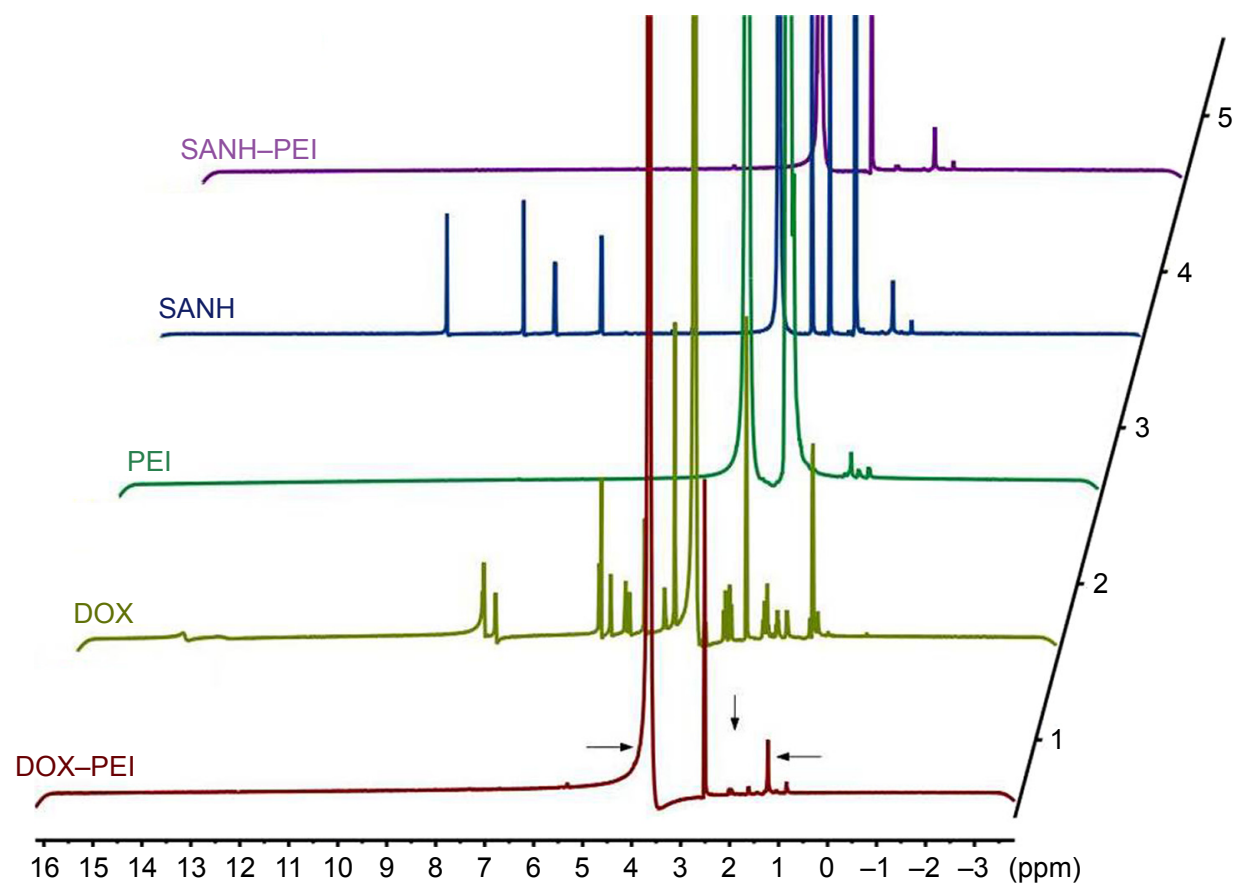

Figure 2 The 'H NMR spectrum of PEI, SANH, SANH-PEI, DOX, DOX-PEI.

Abbreviations: DOX, doxorubicin; NMR, nuclear magnetic resonance; PEl, polyethyleneimine; SANH, succinimidyl 6-hydrazinonicotinate acetone hydrazine.

characteristic peak of DOX was weakly obvious, attributing to the high molecular weight of PEI (25 kDa) comparable to that of DOX (579.98). However, the characteristic peak at $4.0 \mathrm{ppm}$ (the hydrogen of methoxy group of DOX) and the multiple peaks at 1.2 and $2.0 \mathrm{ppm}$ (the characteristic peaks of DOX) in the spectrum of DP could demonstrate the successful conjugation of DOX and PEI. The amount of DOX in DP was $5.77 \% \pm 0.08 \%$, which was determined by using the UV-vis spectrophotometry analysis.

\section{$\mathrm{pH}$-sensitive release of DOX}

An ideal co-delivery system should be able to control the release of the incorporated therapeutics, herein a gene and a chemotherapeutic drug. Moreover, it is evident that hydrazone bond favorably cleaves under low $\mathrm{pH}$ conditions. ${ }^{7}$ Hence, we investigated the in vitro drug release in PBS buffers with different $\mathrm{pH}$ values using the dialysis method. The $\mathrm{pH}$ values were selected as they mimic the $\mathrm{pH}$ of blood plasma ( $\mathrm{pH}$ 7.4) and lysosomal microenvironment in the tumor $(\mathrm{pH} 4-6){ }^{31}$

In vitro release results (Figure 3 ) showed that the DP polymer exhibited the long-term release behavior and $\mathrm{pH}$-response release specifically at the $\mathrm{pH}$ value mimicking the tumor environment. At pH 5.5, approximately $40 \%$ of DOX was released from DP within $280 \mathrm{~h}$, which was much higher than that of its release in $\mathrm{pH}$ 7.4. Based on these results, DP could keep stable in normal $\mathrm{pH}$ environment (blood plasma), which would decrease the leakage of drugs in vivo and improve the drug release performance in the tumor microenvironment.

\section{Preparation and characterization of BMs/ DP/siRNA nanocomplexes}

Cationic DP condensed the anionic siRNA molecules and negatively charged with BMs based on electrostatic interaction, which resulted in $\mathrm{BMs} / \mathrm{DP} / \mathrm{siRNA}$ nanocomplexes. Hence, the use of PEI as a cross-linker provided a basis to co-deliver DOX and siRNA, which could be successfully delivered by escaping the lysosome via proton sponge effect. $^{32}$ To investigate the encapsulation efficiency of nanocomplexes, different DP/siRNA weight ratios and $\mathrm{BMs} / \mathrm{siRNA}$ ratios in $\mathrm{BMs} / \mathrm{DP} / \mathrm{siRNA}$ nanocomplexes were optimized by the agarose gel electrophoresis method as well as DLS detection (Figure 4). As shown in Figure 4A, the fraction of free siRNA was visualized at the low ratio and the band gradually decreased to no color with the increase in DP/siRNA ratio, indicating that siRNA was successfully bound to DP/BMs complex via electrostatic interactions at a DP/siRNA weight ratio of higher than 1.03 .

The size and zeta potential of $\mathrm{BMs} / \mathrm{DP} / \mathrm{siRNA}$ were critical parameters to be evaluated for exploring their cellular uptake efficiency. As shown in Figure 4B and C, the size distribution by the intensity of nanocomplex decreased from 


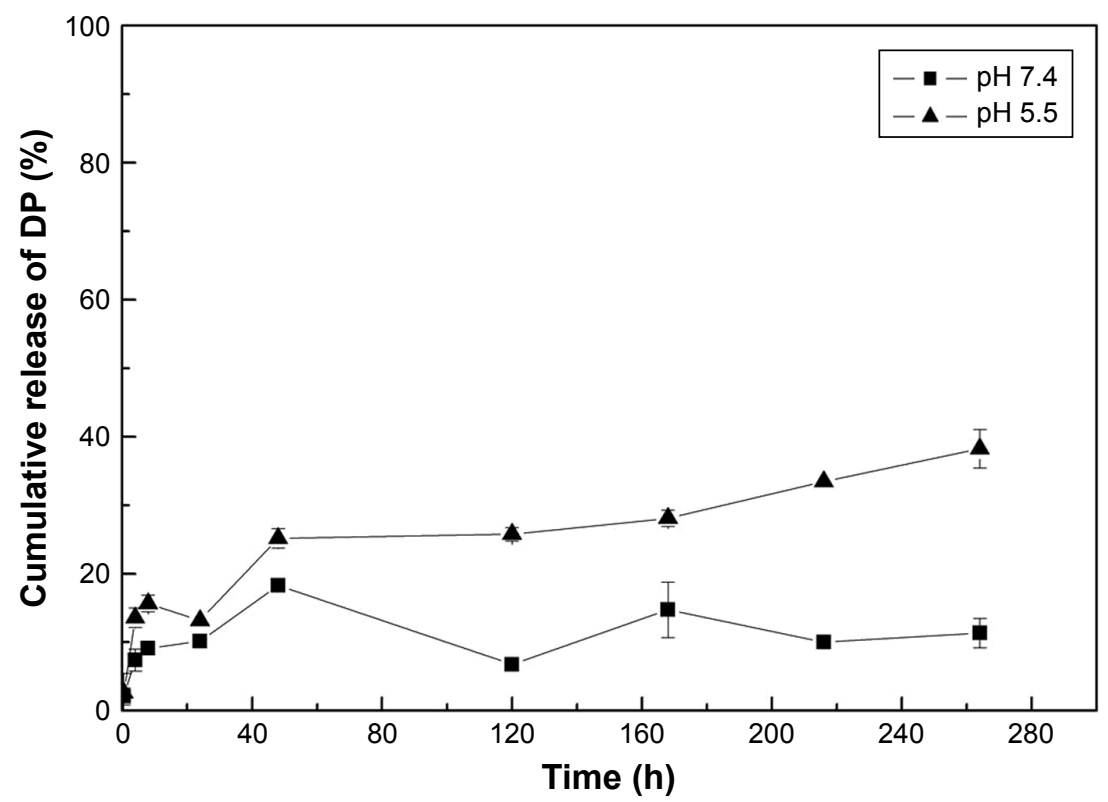

Figure 3 The cumulative release of DP in PBS buffers adjusted to $\mathrm{pH} 5.5$ and 7.4. Abbreviation: DP, doxorubicin-polyethyleneimine.

325 to $118 \mathrm{~nm}$ with the increase in DP/siRNA ratios from 1.03 to 2.58 , indicating that unbound siRNA was condensed to form much more compact nanocomplexes. Moreover, the zeta potential values of $\mathrm{BMs} / \mathrm{DP} / \mathrm{siRNA}$ nanocomplexes were in the range of 29 and $48 \mathrm{mV}$ and with excellent dispersion stability. Multiple studies showed that nanoparticle within $200 \mathrm{~nm}$ could promote the accumulation due to enhanced permeability and retention effect in tumor tissue. ${ }^{13}$ In addition, a modest positive potential would significantly increase the interactions with the negatively charged cell membrane. ${ }^{33}$ Therefore, DP/siRNA ratio of 2.06 was selected as the optimal value for further studies.

To obtain the optimal content of BMs, the gel images of BMs/DP/siRNA complexes with different BMs/siRNA weight ratios were investigated (Figure 4D). It is evident from the results that the free siRNA could not be observed, indicating that the amounts of BMs had a little effect on the formation of nanocomposites. From Figure $4 \mathrm{E}$ and $\mathrm{F}$, when the BMs/siRNA weight ratio was 1:5, the size (distribution by intensity) was relatively small $(107 \mathrm{~nm})$ and the potential value was positive $(31.1 \pm 1.01 \mathrm{mV})$, which was significantly advantageous to augment the contact of nanocomplexes with the cells and increase their cellular internalization efficiency.

\section{Stability of BMs/DP/siRNA nanocomplexes} As shown in Figure 5A, the siRNA from BMs/DP/siRNA composites was released when mixed with heparin, which might be due to the stronger interaction of heparin with the nanocomplexes. ${ }^{34}$ Moreover, at the heparin/siRNA weight ratio of 100 and incubation time of $15 \mathrm{~min}$, the siRNA was completely dissociated from the complex and resulted in the formation of a gel.

To evaluate the stability in physiological fluids, nanocomplexes were incubated in 50\% FBS (Figure 5B). Noticeably, the naked siRNA was degraded within 60 min while $\mathrm{BMs} / \mathrm{DP} / \mathrm{siRNA}$ complex could still survive for $150 \mathrm{~min}$. Moreover, the enzyme stability assay in Figure 5C also showed that the BMs/DP/siRNA complex could slow down the degradation of siRNA. Above all, the new combination delivery system could protect siRNA from degradation and increase the circulation time.

\section{Cell viability assay}

In our previous study, the BMs group maintained a high level of cell viability throughout the incubation period for 7 days based on the CCK-8 assay. ${ }^{35}$ Here, the formation of BMs/DP/ siRNA nanocomplexes was achieved through electrostatic interactions involving multi-step chemical synthesis. More importantly, the prepared complexes have no ketone bonds, which might be the reason for its stability. Therefore, it is required to assess the cell viability of nanocomplexes. In a dose-dependent assay, the viability of cells in the blank vector BMs-PEI/SANH group was higher than $80 \%$, indicating that the blank vector had no significant inhibitory effect on HeLa cells (Figure 6A). And the inhibition rate of BMs/ DP/siRNA (STAT 3) complex on cell proliferation was significantly higher than that of naked siRNA. Moreover, the inhibitory effect of co-delivery of siRNA and DOX was 
A

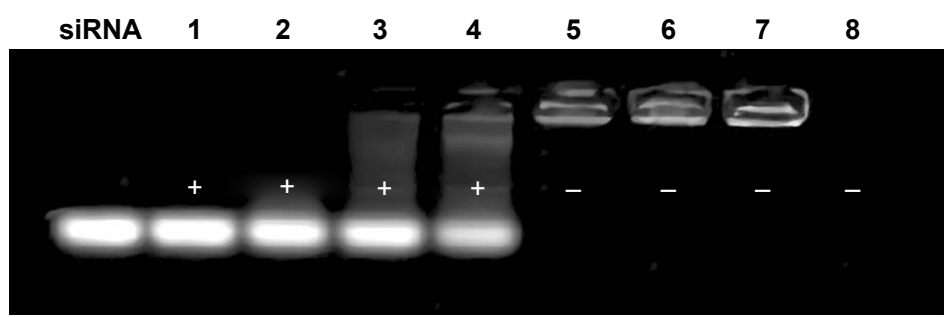

B

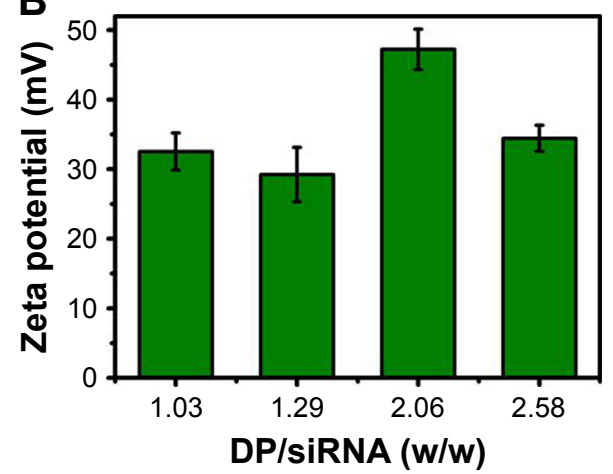

C

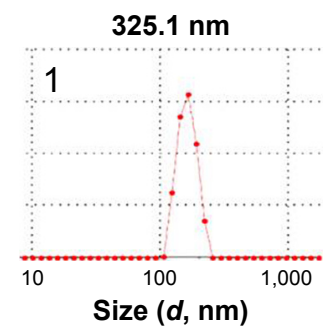

D

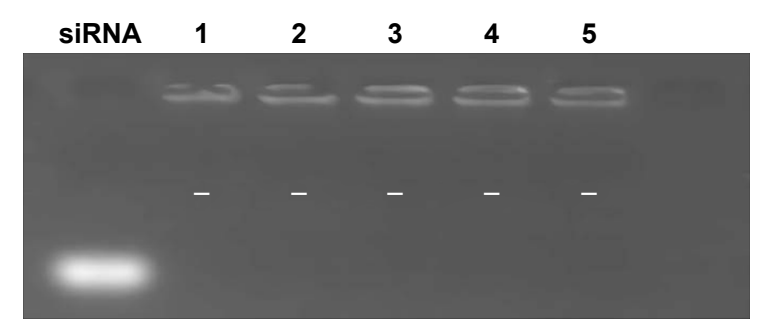

$253.5 \mathrm{~nm}$

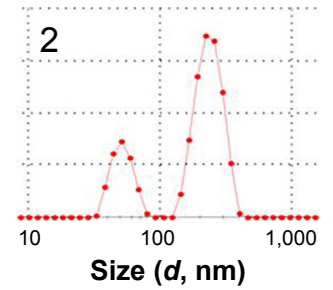

Size $(d, \mathrm{~nm})$
$164.4 \mathrm{~nm}$

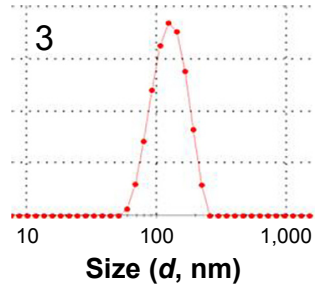

$118.4 \mathrm{~nm}$

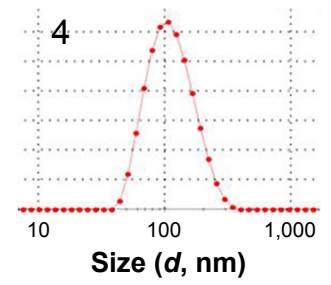

E

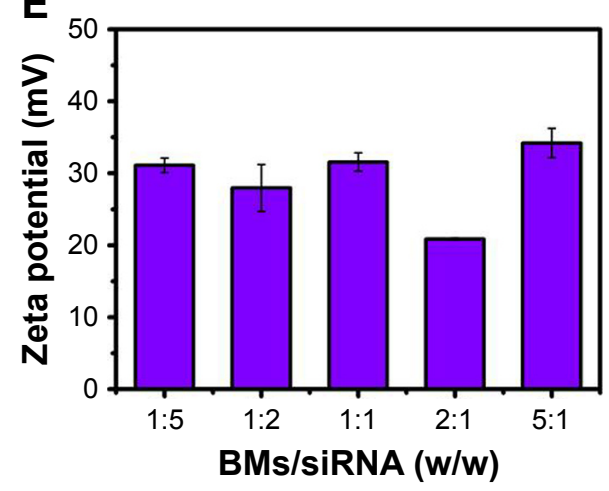

$\mathbf{F}$

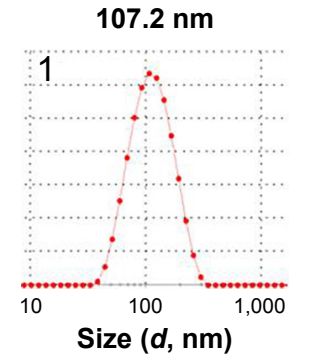

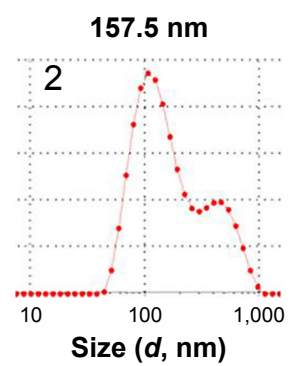
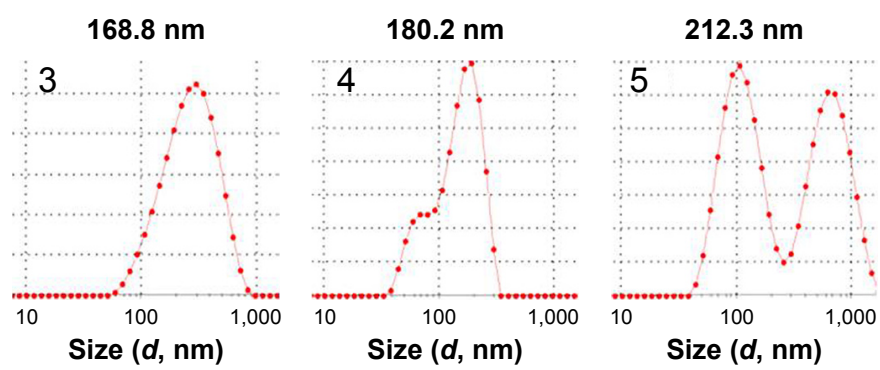

Figure 4 The agarose gel electrophoresis results and characterization of BMs/DP/siRNA complexes

Notes: (A) Gel electrophoresis results of BMs/DP/siRNA complexes with different DP/siRNA weight ratios, I-8: DP/siRNA ratio - 0, 0.13, 0.26, 0.52, I.03, I.29, 2.06, and 2.58, (B) Zeta potential results of BMs/DP/siRNA complexes with different DP/siRNA weight ratios. (C) Diameter results (intensity) of BMs/DP/siRNA complexes with different DP/siRNA ratios, I-4: DP/siRNA ratio - I.03, 1.29, 2.06, and 2.58. (D) Gel images of BMs/DP/siRNA complexes with different BMs/siRNA weight ratios, I-5: BMs/siRNA weight ratio - I:5, I:2, I:I, 2:I, and 5:I. (E) Zeta potential results of BMs/DP/siRNA complexes with different BMs/siRNA weight ratios. (F) Diameter results (intensity) of BMs/DP/siRNA complexes with different BMs/siRNA ratios, I-5: BMs/siRNA weight ratio - I:5, I:2, I:1, 2:I, and 5:1.

Abbreviations: BMs, bacterial magnetosomes; DP, doxorubicin-polyethylenimine.

higher than that of BMs-PEI/SANH-siRNA (STAT 3), which showed that the combination system resulted in the excellent synergistic therapeutic effect. The dose of 7.5 pmol was chosen for further investigation when the DP/siRNA ratio was 2.06 , BMs/siRNA ratio was $1: 5$, the size was relatively small, and potential was positive. Therefore, BMs/DP/siRNA complexes could play a synergistic antitumor effect of gene therapy with chemotherapy simultaneously.

As shown in Figure 6B, cell viabilities of most samples showed an upward trend after the transient decline, which 
A

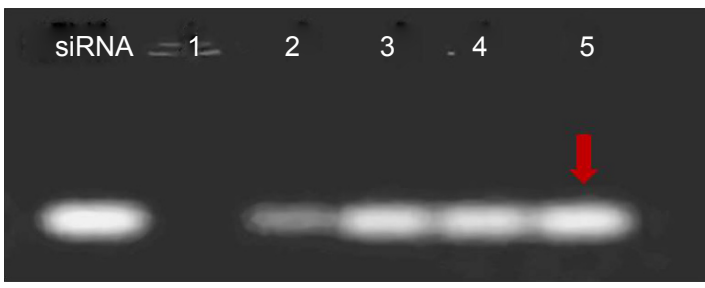

B

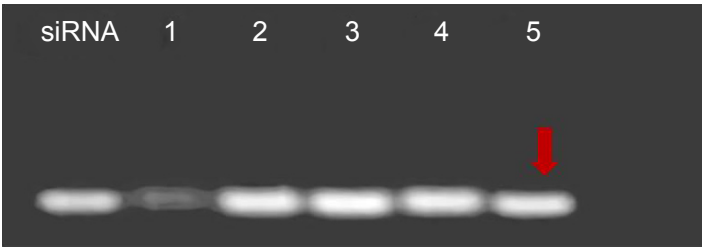

C

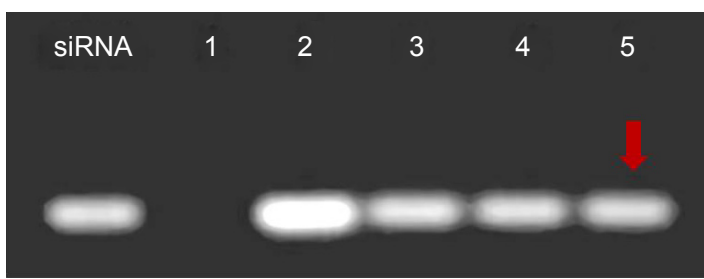

Figure 5 Agarose gel electrophoresis assay of BMs/DP/siRNA complexes. Notes: (A) The heparin decomplexation assay of BMs/DP/siRNA. I: BMs/DP/siRNA and 2-5: heparin/siRNA weight ratio was 2, 10, 25, and 100. (B) The serum stability of BMs/DP/siRNA. I: Naked siRNA incubation with $50 \%$ serum for $60 \mathrm{~min}$ and 2-5: $\mathrm{BMs} / \mathrm{DP} / \mathrm{siRNA}$ incubation with $50 \%$ serum for $60,90,120$, and $150 \mathrm{~min}$. (C) The enzyme stability of BMs/DP/siRNA. I: Naked siRNA incubation with RNase A for 60 min and 2-5: BMs/DP/siRNA incubation with RNase A for 60, 90, 120, and $150 \mathrm{~min}$. Abbreviations: BMs, bacterial magnetosomes; DP, doxorubicin-polyethyleneimine.

might be that the nanocomplex could not completely inhibit the viability of cells, and the residual quantity was not enough to play the antitumor effect sequentially; thus, survival cells began to proliferate after $48 \mathrm{~h}$ culturing.

\section{A}

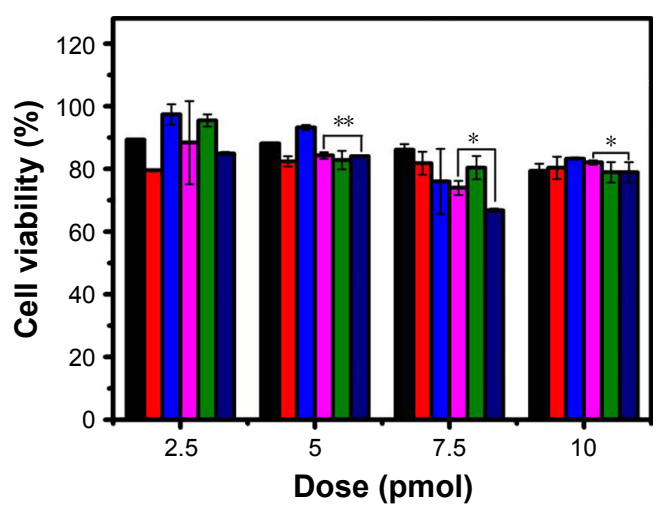

m siRNA $\square$ DOX $\square$ BMs-PEI/SANH

$\square$ BMs-PEI/SANH-siRNA (STAT 3)

$\square$ BMs-PEI/SANH-SiRNA (NC)

- BMs-DP-siRNA (STAT 3)
However, BMs/DP/siRNA had generally the most significant inhibitory effect on cells within $48 \mathrm{~h}$. Therefore, it could be concluded that the combination strategy performed a better synergistic efficiency.

\section{Cell apoptosis by using AO/EB dual staining assay}

As shown in Figure 7, the negative control group showed almost no obvious apoptosis. However, the siRNA- and DOX-treated cells underwent early and late apoptosis, while the BMs-PEI/SANH-siRNA group resulted in late apoptotic cells, in which the sizes were not uniform and the surface was budding. To this end, BMs/DP/siRNA induced apoptosis to most of the HeLa cells and resulted in the significant chromatin condensation, indicating that the co-delivery (BMs/DP/siRNA) yielded a synergistic antitumor efficiency over monotherapy (BMs-PEI/SANH-siRNA).

\section{Cellular uptake of BMs/DP/siRNA nanocomplexes}

The delivery of active siRNA to the desired site area has remained as one of the main obstacles during the development of siRNA-based delivery platforms as only a small amount of active siRNA was delivered, and the remaining was degraded. So we examined the intracellular distribution of new co-delivery nanocomplexes by using CLSM through tracking siRNA that labeled with FAM, nucleus by DAPI staining and DP conjugate, which could be traced as DOX itself is an autofluorescence molecule.

B

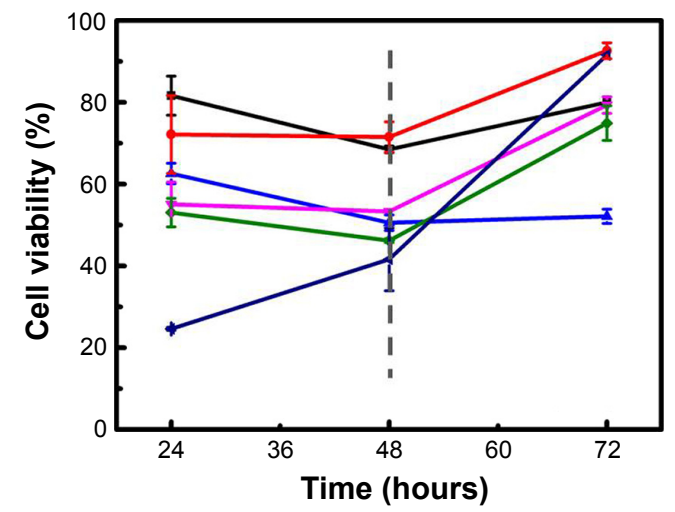

- siRNA $\rightarrow$ DOX $\leftarrow$ BMs-PEI/SANH
- BMs-PEI/SANH-siRNA (STAT 3)
$\rightarrow$ BMs-PEI/SANH-SiRNA (NC)
$\rightarrow$ BMs-DP-siRNA (STAT 3)

Figure 6 Cell viability assay of siRNA, DOX, BMs-PEI/SANH, BMs-PEI/SANH-siRNA (STAT 3), BMs-PEI/SANH-siRNA (NC), and BMs/DP/siRNA (STAT 3) on the growth of HeLa cells ( $10^{5}$ cells/well).

Note: The effects of $(\mathbf{A})$ contents and (B) treatment times of siRNA, DOX, BMs-PEI/SANH, BMs-PEI/SANH-siRNA (STAT 3), BMs-PEI/SANH-siRNA (NC), and BMs/DP/ siRNA (STAT 3 ) on the growth of HeLa cells $(* P<0.05 ; * * P<0.0$ I).

Abbreviations: BMs, bacterial magnetosomes; DOX, doxorubicin; DP, DOX-PEl; PEI, polyethyleneimine; NC, negative control; SANH, succinimidyl 6-hydrazinonicotinate acetone hydrazine. 

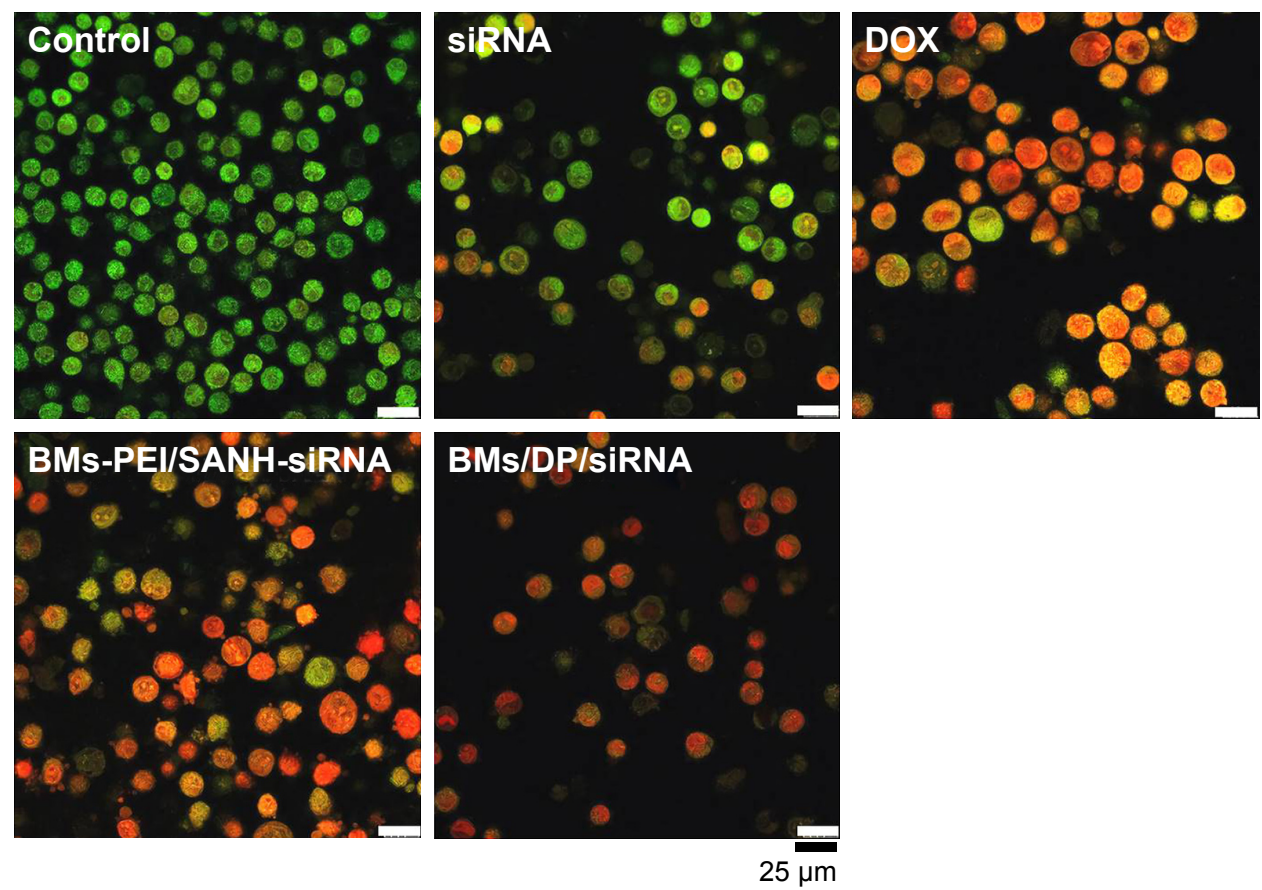

Figure 7 AO/EB-stained images of HeLa cells after culturing with fresh medium (control group), siRNA, DOX, BMs-PEI/SANH-siRNA, and BMs/DP/siRNA for $48 \mathrm{~h}$. Abbreviations: AO, acridine orange; BMs, bacterial magnetosomes; DOX, doxorubicin; DP, DOX-PEl; EB, ethidium bromide; PEI, polyethyleneimine; SANH, succinimidyl 6-hydrazinonicotinate acetone hydrazine.

As shown in Figure 8, the location of siRNA ${ }^{\mathrm{FAM}}$ (green) and DOX-PEI (red) of the nanocomplexes was visualized in the proximity of the nucleus of HeLa cells, which was slightly different from the results of biodistribution in the most previous studies..$^{36}$ The reason behind this phenomenon might be the change in dynamics and distribution of the nanocomplexes due to BMs. Moreover, it is evident that the signs of cleavage of cell nucleus were appeared, indicating that the cells began to apoptosis due to $\mathrm{pH}$-responsive release of DOX by cleaving hydrazone bond, ${ }^{37}$ utilization of PEI, which could avoid the retention of lysosome and increased drug concentration in the cell. ${ }^{38}$ Such a system played an important role in combination therapy, siRNA could play gene silencing effect targeted mRNA in the cell nucleus
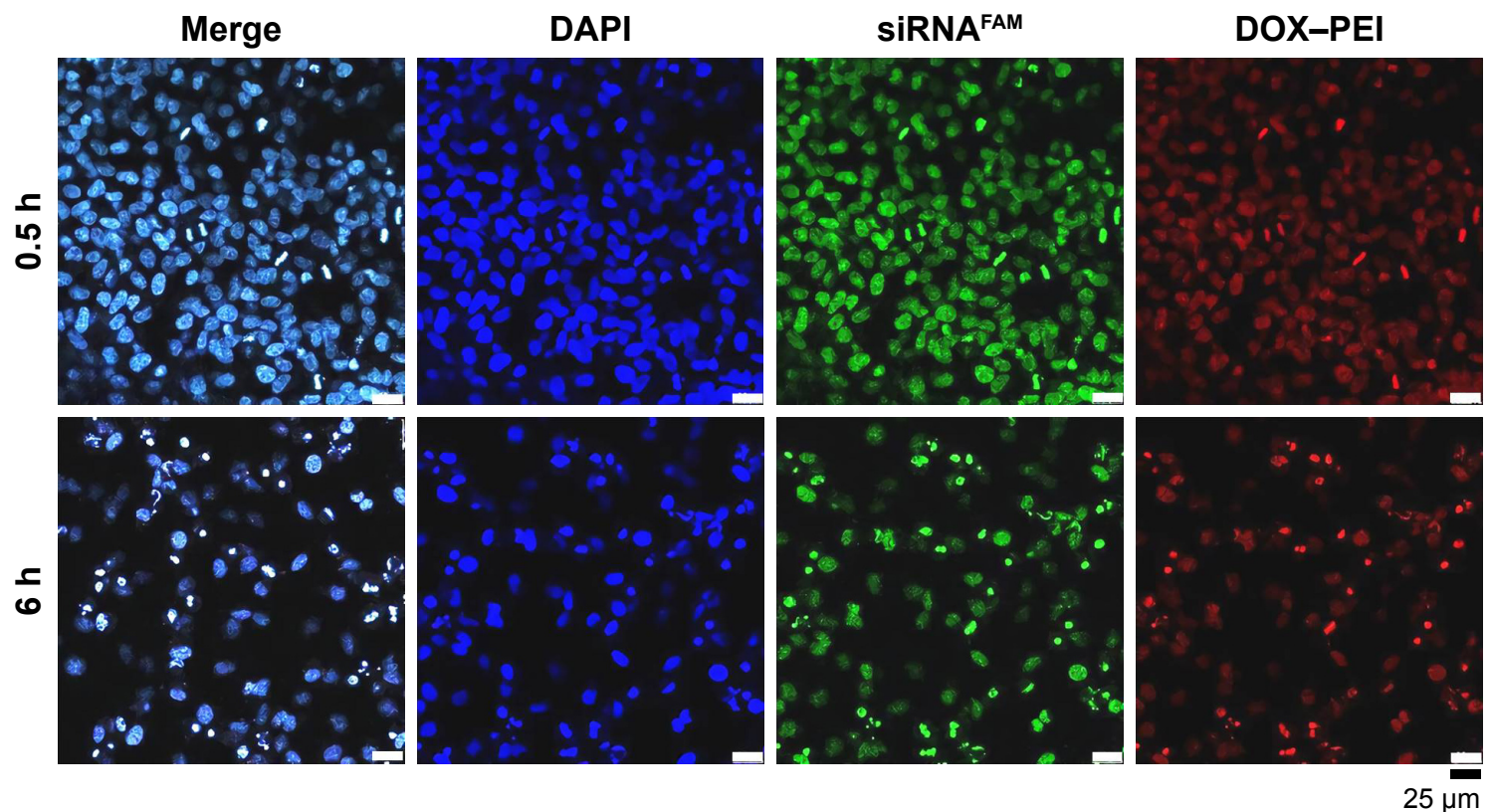

Figure 8 Cellular uptake of BMs/DP/siRNA composites by CLSM when incubating for 0.5 and $6 \mathrm{~h}$.

Abbreviations: BMs, bacterial magnetosomes; CLSM, confocal laser scanning microscope; DOX, doxorubicin; DP, DOX-PEI; PEI, polyethyleneimine. 
and DOX embedded into DNA resulting in the synergistic therapeutic effect within $6 \mathrm{~h}$ of incubation. The co-delivery system prepared in this study provided new evidence for new RNA interference mechanism and laid the foundation for excellent synergistic therapy.

\section{Conclusion}

$\mathrm{BMs} / \mathrm{DP} / \mathrm{siRNAs}$ were successfully constructed through electrostatic interactions between cationic DP with siRNA and BMs. In vitro tests illustrated that BMs/DP conjugate with hydrazone bond provided the $\mathrm{pH}$-response release behavior in a sustained fashion. Moreover, the optimal $\mathrm{BMs} / \mathrm{DP} / \mathrm{siRNA}$ could protect siRNA from degradation in serum and RNase A. Particularly, the designed BMs/DP/ siRNA system achieved an excellent synergistic efficacy compared to that of siRNA alone, which might be a promising therapeutic strategy for disease treatment. However, further investigations relevant to anticancer efficacy in vivo including acute toxicity test, immune toxicity test, metabolic distribution, and tumor inhibition as well as related protein expression are required to be evaluated. Overall, the study has initially demonstrated that the pre-designed BMs/DP/siRNA nano-carrier is promising for co-delivery of a $\mathrm{pH}$-sensitive chemotherapeutic agent and stable siRNA to cancer cells, and could be utilized for superior therapeutic response via combination therapy.

\section{Acknowledgments}

Financial support from National marine economic innovation and development project (16PYY007SF17), the Science Research Foundation of National Health and Family Planning Commission of PRC \& United Fujian Provincial Health and Education Project for Tacking the Key Research (WKJ2016-2-22), the Program for New Century Excellent Talents in Fujian Province University (2014FJ-NCET-ZR01), and the Promotion Program for Young and Middle-aged Teachers in Science and Technology Research of Huaqiao University (ZQN-PY108) is gratefully acknowledged. This research was also supported by the Program for Innovative Research Team in Science and Technology in Fujian Province University. In addition, we must thank Ranjith Kumar Kankala and Mingzhi Zhu (University of Huaqiao University) for the revision and suggestions of our manuscript.

\section{Disclosure}

The authors report no conflicts of interest in this work.

\section{References}

1. Gandhi NS, Tekade RK, Chougule MB. Nanocarrier mediated delivery of siRNA/miRNA in combination with chemotherapeutic agents for cancer therapy: current progress and advances. J Control Release. 2014; 194:238-256.

2. Liu S, Guo Y, Huang R, et al. Gene and doxorubicin co-delivery system for targeting therapy of glioma. Biomaterials. 2012;33(19):4907-4916.

3. Pugazhendhi A, Edison TNJI, Velmurugan BK, Jacob JA, Karuppusamy I. Toxicity of Doxorubicin (Dox) to different experimental organ systems. Life Sci. 2018;200:26-30.

4. Deng ZJ, Morton SW, Ben-Akiva E, Dreaden EC, Shopsowitz KE, Hammond PT. Layer-by-layer nanoparticles for systemic codelivery of an anticancer drug and siRNA for potential triple-negative breast cancer treatment. ACS Nano. 2013;7(11):9571-9584.

5. Oh HR, Jo HY, Park JS, et al. Galactosylated Liposomes for Targeted Co-Delivery of Doxorubicin/Vimentin siRNA to Hepatocellular Carcinoma. Nanomaterials (Basel). 2016;6(8):141.

6. Kang SS, Cho HA, Kim JS. Biodistribution and improved anticancer effect of NIK-siRNA in combination with 5-FU for hepatocellular carcinoma. Arch Pharm Res. 2011;34(1):79-86.

7. Xu C, Tian H, Sun H, Jiao Z, Zhang Y, Chen X. A pH sensitive co-delivery system of siRNA and doxorubicin for pulmonary administration to B16F10 metastatic lung cancer. RSC Adv. 2015;5(125):103380-103385.

8. Xu PY, Kankala RK, Pan YJ, Yuan H, Wang SB, Chen AZ. Overcoming multidrug resistance through inhalable siRNA nanoparticles-decorated porous microparticles based on supercritical fluid technology. Int $J$ Nanomedicine. 2018;13:4685-4698.

9. Kankala RK, Lin X-F, Song H-F, et al. Supercritical Fluid-Assisted Decoration of Nanoparticles on Porous Microcontainers for Codelivery of Therapeutics and Inhalation Therapy of Diabetes. ACS Biomater Sci Eng. 2018.

10. Dong DW, Xiang B, Gao W, Yang ZZ, Li JQ, Qi XR. pH-responsive complexes using prefunctionalized polymers for synchronous delivery of doxorubicin and siRNA to cancer cells. Biomaterials. 2013;34(20): 4849-4859.

11. Meng H, Mai WX, Zhang H, et al. Codelivery of an optimal drug/siRNA combination using mesoporous silica nanoparticles to overcome drug resistance in breast cancer in vitro and in vivo. ACS Nano. 2013;7(2): 994-1005.

12. Kankala RK, Tsai PY, Kuthati Y, Wei PR, Liu CL, Lee CH. Overcoming multidrug resistance through co-delivery of ROS-generating nano-machinery in cancer therapeutics. J Mater Chem B. 2017;5(7): $1507-1517$.

13. Kankala RK, Liu CG, Chen AZ, et al. Overcoming Multidrug Resistance through the Synergistic Effects of Hierarchical pH-Sensitive, ROS-Generating Nanoreactors. ACS Biomater Sci Eng. 2017;3(10): 2431-2442.

14. Mujokoro B, Adabi M, Sadroddiny E, Adabi M, Khosravani M. Nanostructures mediated co-delivery of therapeutic agents for glioblastoma treatment: A review. Mater Sci Eng C Mater Biol Appl. 2016;69: 1092-1102.

15. Ganas C, Weiß A, Nazarenus M, et al. Biodegradable capsules as nonviral vectors for in vitro delivery of PEI/siRNA polyplexes for efficient gene silencing. J Control Release. 2014;196:132-138.

16. Xie L, Tan Y, Wang Z, et al. ع-Caprolactone-modified polyethylenimine as efficient nanocarriers for siRNA delivery in vivo. ACS Appl Mater Interfaces. 2016;8(43):29261-29269.

17. Han YH, Kankala RK, Wang SB, Chen AZ. Leveraging engineering of indocyanine green-encapsulated polymeric nanocomposites for biomedical applications. Nanomaterials (Basel). 2018;8(6):E360.

18. Suo A, Qian J, Xu M, Xu W, Zhang Y, Yao Y. Folate-decorated PEGylated triblock copolymer as a $\mathrm{pH} /$ reduction dual-responsive nanovehicle for targeted intracellular co-delivery of doxorubicin and Bcl-2 siRNA. Mater Sci Eng C Mater Biol Appl. 2017;76:659-672. 
19. Sun J, Li Y, Liang XJ, Wang PC. Bacterial magnetosome: a novel biogenetic magnetic targeted drug carrier with potential multifunctions. J Nanomater. 2011;2011(2011):469031-469043.

20. Liu YG, Dai QL, Wang SB, Deng QJ, Wu WG, Chen AZ. Preparation and in vitro antitumor effects of cytosine arabinoside-loaded genipin-poly-1-glutamic acid-modified bacterial magnetosomes. Int $J$ Nanomedicine. 2015;10:1387-1397.

21. Schüler D. Formation of magnetosomes in magnetotactic bacteria. J Mol Microbiol Biotechnol. 1999;1(1):79-86.

22. Liu Y, Xie M, Wang S, Zheng Q, Chen A, Deng Q. Facile fabrication of high performances MTX nanocomposites with natural biomembrane bacterial nanoparticles using GP. Mater Lett. 2013;100: 248-251.

23. Guo L, Huang J, Zheng LM. Control generating of bacterial magnetic nanoparticle-doxorubicin conjugates by poly-L-glutamic acid surface modification. Nanotechnology. 2011;22(17):175102.

24. Tang YS, Wang D, Zhou C, et al. Bacterial magnetic particles as a novel and efficient gene vaccine delivery system. Gene Ther. 2012;19(12): 1187-1195.

25. Han L, Li S, Yang Y, Zhao F, Huang J, Chang J. Comparison of magnetite nanocrystal formed by biomineralization and chemosynthesis. J Magn Magn Mater. 2007;313(1):236-242.

26. Xiao Y, Jaskula-Sztul R, Javadi A, et al. Co-delivery of doxorubicin and siRNA using octreotide-conjugated gold nanorods for targeted neuroendocrine cancer therapy. Nanoscale. 2012;4(22):7185-7193.

27. Liu C, Liu F, Feng L, Li M, Zhang J, Zhang N. The targeted co-delivery of DNA and doxorubicin to tumor cells via multifunctional PEI-PEG based nanoparticles. Biomaterials. 2013;34(10):2547-2564.

28. Kievit FM, Wang FY, Fang C, et al. Doxorubicin loaded iron oxide nanoparticles overcome multidrug resistance in cancer in vitro. J Control Release. 2011;152(1):76-83.
29. Wang M, Liu T, Han L, Gao W, Yang S, Zhang N. Functionalized O-carboxymethyl-chitosan/polyethylenimine based novel dual $\mathrm{pH}$ responsive nanocarriers for controlled co-delivery of DOX and genes. Polym Chem. 2015;6(17):3324-3335.

30. Lin JT, Zou Y, Wang C, et al. Cationic micellar nanoparticles for DNA and doxorubicin co-delivery. Mater Sci Eng C Mater Biol Appl. 2014;44: 430-439.

31. Guo M, Yan Y, Liu X, et al. Multilayer nanoparticles with a magnetite core and a polycation inner shell as $\mathrm{pH}$-responsive carriers for drug delivery. Nanoscale. 2010;2(3):434-441.

32. Godbey WT, Mikos AG. Recent progress in gene delivery using nonviral transfer complexes. J Control Release. 2001;72(1-3):115-125.

33. Verma A, Stellacci F. Effect of surface properties on nanoparticle-cell interactions. Small. 2010;6(1):12-21.

34. Moret I, Esteban Peris J, Guillem VM, et al. Stability of PEI-DNA and DOTAP-DNA complexes: effect of alkaline $\mathrm{pH}$, heparin and serum. J Control Release. 2001;76(1-2):169-181.

35. Long R, Liu Y, Dai Q, Wang S, Deng Q, Zhou X. A Natural bacteriumproduced membrane-bound nanocarrier for drug combination therapy. Materials (Basel). 2016;9(11):889.

36. Lin G, Zhu W, Yang L, et al. Delivery of siRNA by MRI-visible nanovehicles to overcome drug resistance in MCF-7/ADR human breast cancer cells. Biomaterials. 2014;35(35):9495-9507.

37. Xiong XB, Ma Z, Lai R, Lavasanifar A. The therapeutic response to multifunctional polymeric nano-conjugates in the targeted cellular and subcellular delivery of doxorubicin. Biomaterials. 2010;31(4):757-768.

38. Lee ES, Na K, Bae YH. Doxorubicin loaded pH-sensitive polymeric micelles for reversal of resistant MCF-7 tumor. J Control Release. 2005; 103(2):405-418.
International Journal of Nanomedicine

\section{Publish your work in this journal}

The International Journal of Nanomedicine is an international, peerreviewed journal focusing on the application of nanotechnology in diagnostics, therapeutics, and drug delivery systems throughout the biomedical field. This journal is indexed on PubMed Central, MedLine, CAS, SciSearch $\AA$, Current Contents $₫ /$ Clinical Medicine,

\section{Dovepress}

Journal Citation Reports/Science Edition, EMBase, Scopus and the Elsevier Bibliographic databases. The manuscript management system is completely online and includes a very quick and fair peer-review system, which is all easy to use. Visit http://www.dovepress.com/ testimonials.php to read real quotes from published authors. 